\title{
Opacity calculation for target physics using the ABAKO/RAPCAL code
}

\author{
E. Mínguez ，R. Florido ，R. Rodríguez ，J.M. Gil ，J.G. Rubiano ， \\ M.A. Mendoza , D. Suárez , P. Martel
}

Instituto de Fusión Nuclear, Universidad Politécnica de Madrid, Madrid 28006, Spain

Departamento de Fisica, Universidad de Las Palmas de Gran Canaria, Las Palmas de Gran Canaria 35017, Spain

\section{A R T I C L E I N F O}

\section{PACS:}

52.25

Keywords:

Opacities

Emissivities

Population kinetics

\begin{abstract}
A B S T R A C T
Radiative properties of hot dense plasmas remain a subject of current interest since they play an important role in inertial confinement fusion (ICF) research, as well as in studies on stellar physics. In particular, the understanding of ICF plasmas requires emissivities and opacities for both hydro-simulations and diagnostics. Nevertheless, the accurate calculation of these properties is still an open question and continuous efforts are being made to develop new models and numerical codes that can facilitate the evaluation of such properties. In this work the set of atomic models ABAKO/RAPCAL is presented, as well as a series of results for carbon and aluminum to show its capability for modeling the population kinetics of plasmas in both LTE and NLTE regimes. Also, the spectroscopic diagnostics of a laser-produced aluminum plasma using ABAKO/RAPCAL is discussed. Additionally, as an interesting application of these codes, fitting analytical formulas for Rosseland and Planck mean opacities for carbon plasmas are reported. These formulas are useful as input data in hydrodynamic simulation of targets where the computation task is so hard that in line computation with sophisticated opacity codes is prohibitive.
\end{abstract}

\section{Introduction}

In several research areas, such as astrophysics and both magnetic and inertial confinement fusions [1], the knowledge of the interaction between the radiation and plasma is essential. In these plasmas the calculation of its radiative properties is a difficult task due to the huge number of levels and transitions involved, even for low $Z$ elements. In particular, the understanding of ICF plasmas requires emissivities and opacities for both hydro-simulations and diagnostics [2].

During the last two decades, carbon and aluminum plasmas have been of particular interest for experimental and theoretical work [3-6]. Most of this work was developed under LTE conditions, and since the calculation of plasma radiative properties is very complex, this is a welcome simplification. However, to assist in the calculation of such properties beyond the LTE assumption, the code ABAKO/RAPCAL has been developed [7-9], which can determine populations, radiative properties, spectrally resolve opacities and emissivities, and perform K-shell spectroscopic diagnostics to infer both the average electron density and the temperature of the plasma in LTE and non-LTE (NLTE) regimes.
The atomic structure model used in this code follows a relativistic detailed-configuration-accounting (DCA) scheme, where relativistic electronic configurations are determined by solving the monoelectronic Dirac equation, using parametric analytical potentials developed by our group in the last years. The non-local thermodynamic equilibrium (NLTE) system of rate equations is solved assuming steady-state, although work is in progress to deal with time-dependent situations. To compute the rate coefficients it mostly uses well-known analytical formulas and it has been demonstrated that with these rate coefficients, the code provides good results in a wide range of temperatures and densities, reproducing the Coronal and Saha results in the limits of low and high densities, respectively. Optically thin and thick plasmas can also be treated.

This work provides a preliminary scope of applications of this code. First, a theoretical analysis of carbon and aluminum plasmas is performed covering a wide range of temperatures and densities. Some results are compared with those from other theoretical models. Also, the computer code has been recently improved to provide spectrally resolved intensities and a first experimental application to an ultra-dense hot aluminum plasma is discussed.

\section{Computational model}

The calculations and analysis carried out in this work have been made following the ABAKO/RAPCAL flowchart described in Fig. 1. 
The computational model is basically composed of three modules: atomic structure, level populations and optical properties. However, it also permits the use of external modules providing atomic data which are mainly used for diagnostic purposes.

\subsection{Atomic structure module}

The atomic structure model can work using two levels of description depending on the atomic number or the ionization degree of the element under consideration. For lowly ionized intermediate- and high- $Z$ plasmas, it works using a set of analytical potentials, which can model both isolated $[10,11]$ and immersed ions into plasmas [12], including plasma effects and single- and core-excited configurations [13]. For situations where faster calculations are required, the code can obtain the atomic data using a relativistically screened hydrogenic model [14].

For low-Z or highly ionized, medium- and high- $Z$ plasmas it uses a detailed level description provided by external atomic data. At present, the external atomic data are generated using the FAC code [15], which is designed to provide atomic data in the detailed level accounting (DLA) approach. The energy of the levels of an atomic ion with $N$ electrons is obtained by diagonalizing the relativistic Hamiltonian. The basis states which are usually referred to as configuration state functions (CSFs) are built as anti-symmetric sums of products of $N$ one-electron Dirac spinors. In coupling the angular momenta the standard $j i$-coupling scheme is used. Finally, the approximate atomic wave functions are evaluated by mixing the basis states with the same symmetries, with the mixing coefficients obtained from diagonalizing the total Hamiltonian. The FAC code can also work in the DCA approach by means of configuration averages of the detailed levels.

\subsection{Collisional-radiative module}

The aim of this module is to obtain the charge state and level population distribution using a collisional-radiative (CR) steadystate model. The CR matrix is built and solved level-by-level or configuration-by-configuration depending on whether the DLA or DCA approach is used. The model can be applied to low-to-high Z ions under a wide range of laboratory or astrophysical plasma conditions covering both LTE and NLTE conditions. Special care was taken during the development of the $\mathrm{CR}$ model to achieve an optimal balance between accuracy and computational cost.

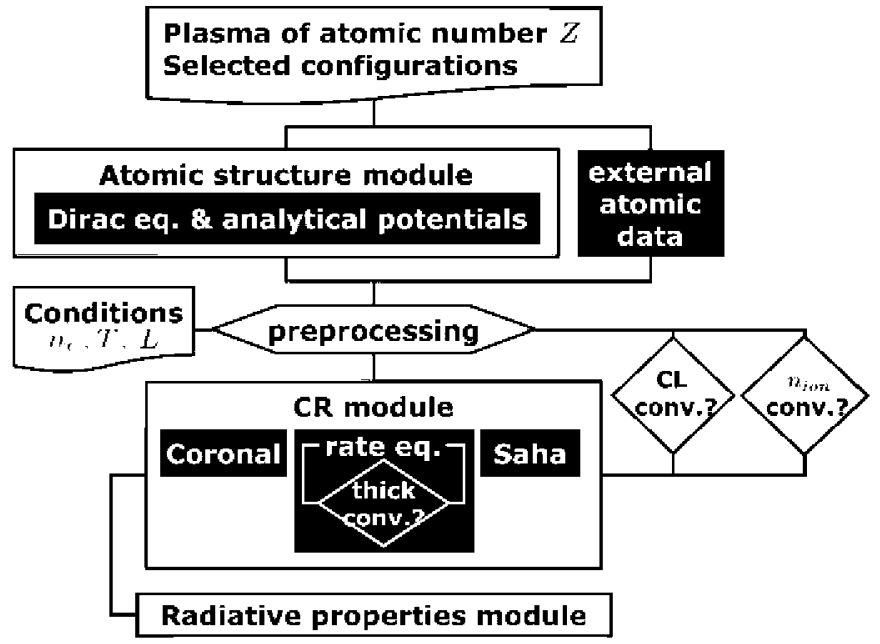

Fig. 1. $A B A K O / R A P C A L$ flowchart
Analytical expressions have been employed to determine the rate coefficients of the atomic processes included in the CR model, which yield a substantial saving of computational requirements, but provide satisfactory results in relation to those obtained from more sophisticated codes and experimental data. The processes included in the $\mathrm{CR}$ model are the following: collisional ionization [16] and three-body recombination, spontaneous decay, collisional excitation [17] and deexcitation, radiative recombination [18], electron capture and autoionization. The rates of the inverse processes are obtained through the detailed balance principle. It is worth pointing out that the autoionizing states are included explicitly, since it has been proved that their contribution is critical in the determination of the ionization balance. The cross-section of the autoionization process is evaluated using the detailed balance principle from the electron capture cross-section. This one is obtained from the collisional excitation cross-section using a known approximation [19].

As was previously stated, ABAKO/RAPCAL is able to work under both DLA and DCA approaches. However, the expressions employed for the rate coefficients were developed in a DCA context. Hence, when they are used in DLA approach they are corrected by means of the corresponding branching ratios.

The ABAKO/RAPCAL code is also able to determine level populations and radiative properties of optically thin and thick carbon plasmas. However, only those atomic processes whose rates are independent of the radiation field intensity are explicitly considered in the model. Thus, to take into account the effects of radiation reabsorption the escape factor formalism is used. This methodology is very well-known and frequently used in plasma spectroscopy. Escape factors are considered in the CR model ABAKO in two ways. Firstly, they enter in the atomic physics calculations giving an effective reduction of the spontaneous decay rate coefficient. Secondly, they appear in the determination of the total emergent line intensity. This avoids the need to perform a simultaneous resolution of radiation transport and kinetic equations. A uniform distribution of emitting atoms, isotropic emission and a slab geometry have been assumed.

In this work high density conditions are also considered. As the density increases, the screening effects due to neighboring electrons and ions modify the energy levels while the degeneracy begins to raise the energy of the free electrons. As a result, there is a change in both the ionization potentials of bound states and the level occupancy numbers which leads to pressure ionization. In spite of the well-known critical importance of pressure ionization in the calculation of ionic abundances and level populations, great

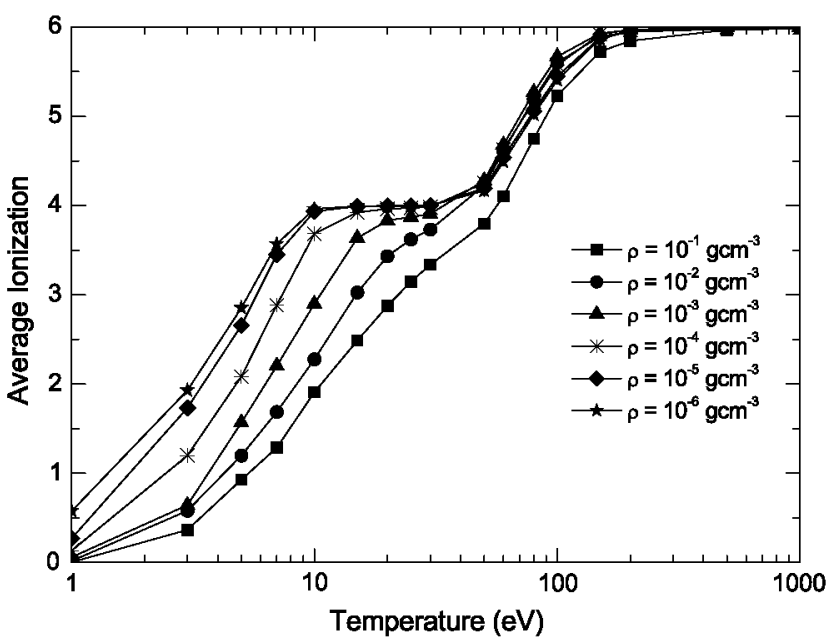

Fig. 2. Average ionization for carbon plasma versus temperature at several densities. 
Table 1

LTE Planck $\left(\kappa_{\mathrm{P}}\right)$ and Rosseland $\left(\kappa_{\mathrm{R}}\right)$ mean opacities $\left(\mathrm{cm}^{2} / \mathrm{g}\right)$ obtained with ABAKO/ RAPCAL and LEDCOP for a carbon plasma at several temperatures $(\mathrm{eV})$ and densities $\left(\mathrm{g} \mathrm{cm}^{-3}\right)$.

\begin{tabular}{lllllll}
\hline$T$ & $\rho$ & \multicolumn{2}{l}{ ABAKO/RAPCAL } & & \multicolumn{2}{l}{ LEDCOP } \\
\cline { 3 - 4 } \cline { 6 - 7 } & & $\kappa_{\mathrm{P}}$ & $\kappa_{\mathrm{R}}$ & & $\kappa_{\mathrm{P}}$ & $\kappa_{\mathrm{R}}$ \\
\hline 10 & $10^{-5}$ & $1.693 \times 10^{3}$ & $1.187 \times 10^{2}$ & & $1.329 \times 10^{3}$ & $1.634 \times 10^{2}$ \\
20 & $10^{-5}$ & $4.330 \times 10^{1}$ & $4.071 \times 10^{0}$ & & $6.315 \times 10^{1}$ & $4.810 \times 10^{0}$ \\
10 & $10^{-3}$ & $4.623 \times 10^{4}$ & $1.758 \times 10^{4}$ & & $5.147 \times 10^{4}$ & $1.808 \times 10^{4}$ \\
20 & $10^{-3}$ & $1.780 \times 10^{3}$ & $2.213 \times 10^{2}$ & & $2.366 \times 10^{3}$ & $3.626 \times 10^{2}$ \\
10 & $10^{-1}$ & $2.354 \times 10^{5}$ & $8.511 \times 10^{4}$ & & $1.699 \times 10^{5}$ & $6.902 \times 10^{4}$ \\
20 & $10^{-1}$ & $2.621 \times 10^{4}$ & $2.503 \times 10^{3}$ & & $3.283 \times 10^{4}$ & $6.573 \times 10^{3}$ \\
\hline
\end{tabular}

difficulties are still found in its modeling and most of the existing CR models and codes take into account the plasma effects in a very approximate way. The effect of the pressure ionization is often described only in terms of a lowering of the ionization potential or continuum-lowering $(\mathrm{CL})$ and the formulation due to Stewart and Pyatt [20] is applied. This formalism is also used in ABAKO/RAPCAL.

\subsection{Radiative properties module}

Radiative properties, such as emissivities, opacities and radiative power losses, have been obtained with the RAPCAL code $[9,21]$ using atomic data and level population provided by the previous modules. The bound-bound spectrum includes all the allowed transitions in the dipole approximation along with all the detailed atomic levels considered. Line profiles incorporate Doppler, natural and electron-impact widths. For the latter a semiempirical formula [22] is used. Complete redistribution is assumed, so the same profile is used for the emission and the absorption processes. For the bound-free spectrum, it evaluates the cross-section in the distorted wave approximation. Finally, for the free-free spectrum, the semiclassical expression of Kramers was employed [23].

\section{Optical properties of carbon plasmas}

Carbon has been chosen for two reasons. The first one is because of its current interest as a major plasma facing wall component in the international thermonuclear experimental reactor (ITER) [24]. It also plays a major role in inertial fusion scenarios [25] and therefore radiation rates from carbon impurities must be known. Furthermore, some laser experiments have been focused on the spectrally resolved emission from hydrocarbon plasmas [26].

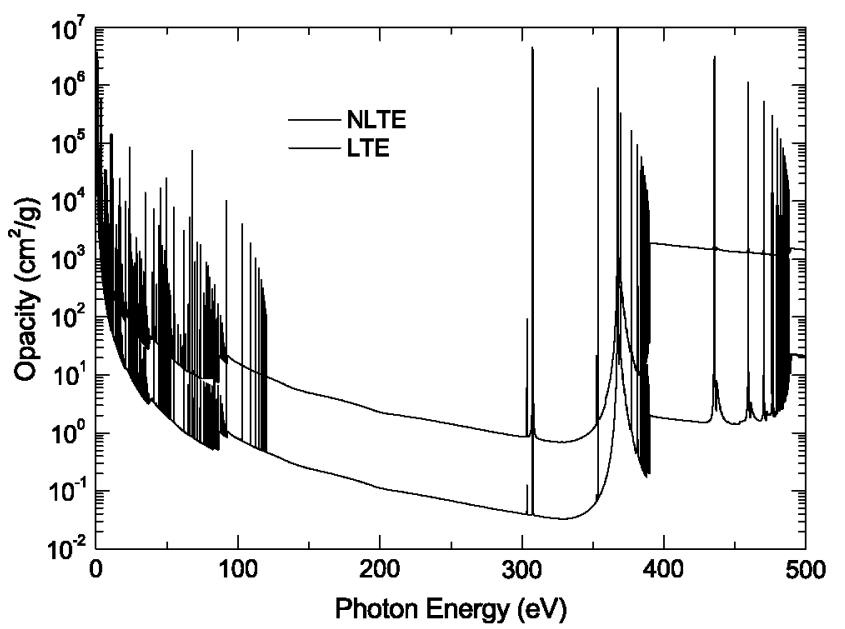

Fig. 3. Spectrally resolved opacity for a carbon plasma at $T=75 \mathrm{eV}$ and $n_{\mathrm{e}}=10^{20} \mathrm{~cm}^{-3}$. Comparison between LTE and NLTE calculations is shown.
Table 2

Average ionization $(\bar{Z})$, Planck $\left(\kappa_{\mathrm{P}}\right)$ and Rosseland $\left(\kappa_{\mathrm{R}}\right)$ mean opacities $\left(\mathrm{cm}^{2} / \mathrm{g}\right)$ for a carbon plasma at $T=75 \mathrm{eV}$ and $n_{\mathrm{e}}=10^{20} \mathrm{~cm}^{-3}$.

\begin{tabular}{llll}
\hline & $\bar{Z}$ & $\kappa_{\mathrm{P}}$ & $\kappa_{\mathrm{R}}$ \\
\hline LTE & 5.919 & $2.895 \times 10^{2}$ & 0.903 \\
NLTE & 4.984 & $5.341 \times 10^{3}$ & 2.153 \\
\hline
\end{tabular}

LTE calculation is shown for comparison.

In recent work [27-29], it has been proved that configuration average (CA) calculations are useful for modeling carbon plasmas at temperatures higher than $10 \mathrm{eV}$. However, for lower temperatures this approach is not accurate enough and a DLA approach is required. Therefore, ABAKO/RAPCAL could be a useful tool to investigate this regime.

\subsection{Spectrally resolved and mean opacities of carbon plasmas in a wide range of densities and temperatures}

The purpose of this section is to show the ability of ABAKO/ RAPCAL to calculate spectrally resolved and mean opacities of optically thin carbon plasmas in a wide range of densities and temperatures, where LTE and NLTE regimes are present. Moreover, changes introduced in the population distribution and radiative properties when the plasma is optically thick are briefly analyzed. Due to the reason commented above, this study is performed following a DLA approach using the atomic data provided externally by the FAC code.
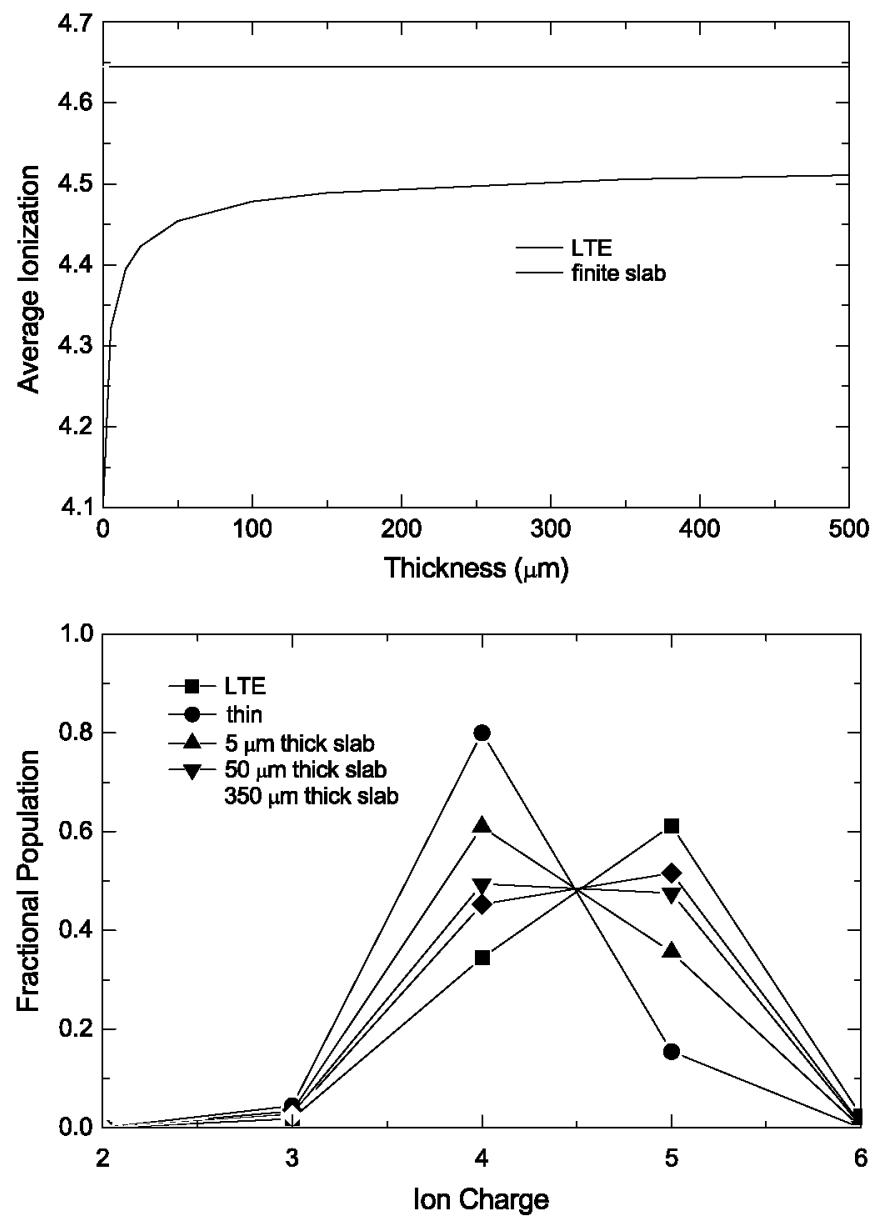

Fig. 4. Analysis of average ionization and charge state distribution for an optically thick carbon plasma at $T=40 \mathrm{eV}$ and $n_{\mathrm{e}}=10^{20} \mathrm{~cm}^{-3}$. Thin case and LTE calculations are also shown for comparison. 
First, the average ionization of a carbon plasma is determined for a wide range of temperatures $(1-1000 \mathrm{eV})$ and densities $\left(10^{-1}\right.$ $10^{-6} \mathrm{~g} \mathrm{~cm}^{-3}$ ) (see Fig. 2). The calculation of average ionization lets us to test the asymptotic behavior of average ionization at low and high densities and allows the determination of the areas in the density-temperature plane that corresponds to the Coronal, LTE and NLTE regimes of optically thin carbon plasma [30].

In order to have a comparison with other sources, Table 1 shows some calculations of the mean opacities (Rosseland and Planck) in LTE obtained with ABAKO/RAPCAL and the LEDCOP code [31] developed at LANL. This code uses a basis set of detailed LS terms including interactions with the plasma which are treated as perturbations. Discrepancies from $3 \%$ at $10 \mathrm{eV}$ and $10^{-3} \mathrm{~g} \mathrm{~cm}^{-3}$, up to $60 \%$ at $20 \mathrm{eV}$ and $10^{-1} \mathrm{~g} \mathrm{~cm}^{-3}$ are found.

With ABAKO/RAPCAL it is possible to calculate the spectrally resolved opacities under LTE and NLTE conditions. To give an example, the opacity for a selected NLTE carbon plasma case at $T=75 \mathrm{eV}$ and $n_{\mathrm{e}}=10^{20} \mathrm{~cm}^{-3}$ has been compared with the LTE calculation (see Fig. 3). For this case, the average ionization and mean opacities are displayed in Table 2 . We observed that there are considerable differences between the calculations. This example demonstrates the importance of determining the population distributions and the spectral quantities with the appropriate model, especially when the plasma density and temperature have genuine non-equilibrium values. Thus, care must be taken to avoid an abuse of the LTE assumption, since it can lead to significant errors.

To analyze the opacity effects in optically thick carbon plasmas, first in (Fig. 4, up) we plotted the average ionization as a function of
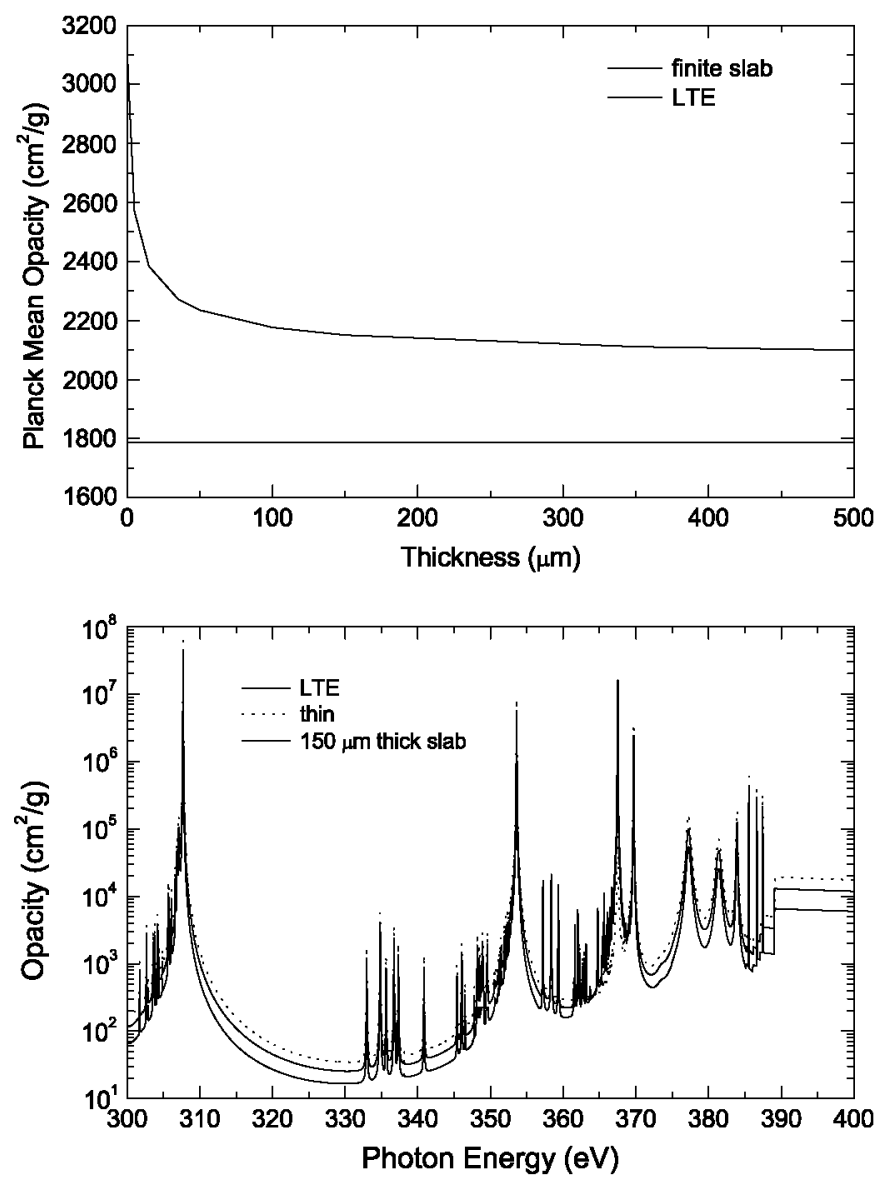

Fig. 5. Planck mean and spectrally resolved opacities of an optically thick carbon plasma at $T=40 \mathrm{eV}$ and $n_{\mathrm{e}}=10^{20} \mathrm{~cm}^{-3}$. Thin case and LTE calculations are also shown for comparison. the slab thickness at fixed conditions of electron density and temperature. The LTE result is also presented for comparison. As expected due to radiation reabsorption, the average ionization increases with the plasma thickness up to the asymptotic limit corresponding to the hypothetical case of an completely opaque plasma for line radiation. Nevertheless the LTE calculation predicts an even higher ionization. This is because, first, opacity effects on continuum radiation processes have not been included, and, second, the calculations have been undertaken with conditions for which the dominant role of collisional processes in the ionization balance is not assured, a requirement for LTE. Changes arising in the charge state distribution are shown in Fig. 4 (down). Of course, the radiation reabsorption also modifies the radiative properties and this is illustrated in Fig. 5 for the Planck mean (up) and spectrally resolved (down) opacities.

\subsection{NLTE analytical mean opacities for carbon plasmas in a wide range of densities and temperatures}

The accurate computation of radiative opacities is needed in ICF target physics analysis. For these targets, 2D radiation hydrodynamic codes are necessary, and the use of thousands of spectrally resolved opacity points for each mesh point takes a large calculation time. The normal procedure consists of averaging these multifrequency opacities into a small number of multigroup opacities. Thus, 2D codes or even 3D codes use opacities for a very small number of groups. In certain cases single-group Rosseland and Planck opacities are used. For this reason, analytical formulas for mean opacities as a function of temperature and density of the plasma are useful.

Analytical expressions can be found in the literature [32-36] for the opacity of low $-Z$ plasmas in a wide range of temperatures and densities.

Now using the ABAKO/RAPCAL code, new analytical expressions can be obtained for carbon plasmas. The expression used as reference to model Rosseland and Planck mean opacities is a power law depending on temperature and density,

$\kappa=\mathrm{e}^{a} T^{b} \rho^{c}$

where $T$ is the temperature (eV) and $\rho$ is the mass density $\left(\mathrm{g} \mathrm{cm}^{-3}\right)$. The definition of the parameter $a$ can change depending on the author.

These expressions are usually fitted to match LTE data but there are no values for NLTE conditions. In a logarithmic representation, Eq. (1) is a plane surface over the entire range of temperatures and densities. This geometrical form can give a good fit for highly ionized plasmas, but it fails at low temperatures where the atomic structure leads to local modulations of the mean opacity.

In order to extend its range of application, Eq. (1) for the Planck mean opacity is modified including a function that depends on the temperature and the electron density,

$\rho \kappa_{\mathrm{P}}=\mathrm{e}^{a_{0}} T^{a_{1}} \rho^{a_{2}} f(x, y)$,

with

$f(x, y)=\mathrm{e}^{a_{3} x y+a_{4} x^{2}+a_{5} y^{2}}$

where $x=\log (T)$ and $y=\log (\rho)$. This new expression is a quadratic fit to the opacity surface which gives more flexibility in relation to a linear one. Fig. 6 (up) shows the Planck mean opacity for carbon plasmas in the range from 1 to $1000 \mathrm{eV}$ from $10^{-10}$ to $10^{-1} \mathrm{~g} \mathrm{~cm}^{-2}$ computed with ABAKO/RAPCAL. A piecewise fit in temperature has been performed due to the complex behavior exhibited by the theoretical opacity surface. Values of the parameters obtained are 

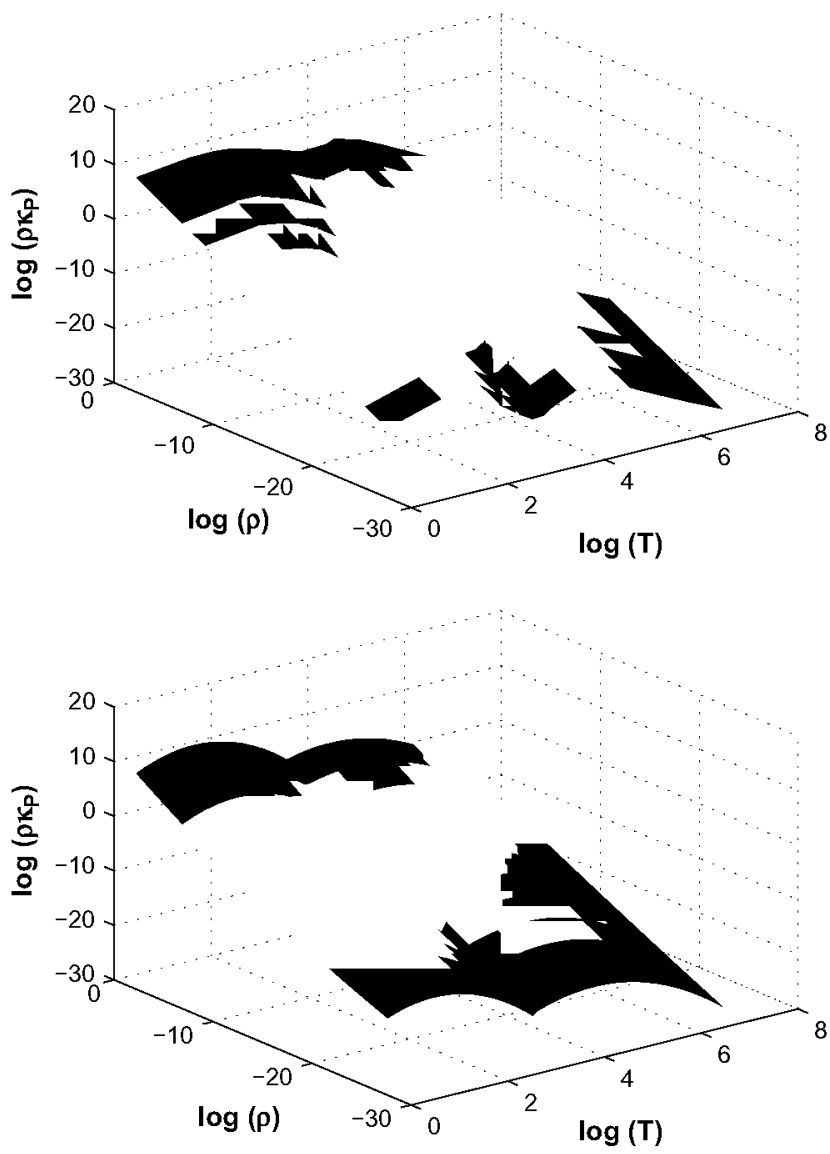

Fig. 6. Planck mean opacity surface for carbon calculated using ABAKO/RAPCAL (up). Fitting surface obtained by application of Eq. (2) (down).

given in Table 3. Figs. 6 (down) and 7 show how the new expression follows the behavior of the theoretical Planck mean opacity over the whole range of temperatures.

\section{Optical properties of aluminum plasmas}

During the past two decades, aluminum plasmas have been of particular interest and much experimental investigation has been undertaken. Theoretically, several studies were carried out to simulate the opacities transmission spectra. However, most of this work has been developed assuming LTE. Furthermore, the theoretical calculation of plasma radiative properties is very complex and it is necessary to use approximations. For these reasons, there is still a lack of complete understanding of these topics and their investigation is still an important area of study. Thus, ABAKO/ RAPCAL is used in this work to study the radiative properties of aluminum plasmas over a wide range of densities and temperatures.

Table 3

Fitting parameters of the analytical formula for Planck mean opacity in an NLTE carbon plasma.

\begin{tabular}{lrr}
\hline & $T \leq 20 \mathrm{eV}$ & $T>20 \mathrm{eV}$ \\
\hline$a_{0}$ & 12.6248 & -12.2285 \\
$a_{1}$ & 4.4268 & 10.6597 \\
$a_{2}$ & 1.3453 & 1.2104 \\
$a_{3}$ & 0.0332 & -0.0309 \\
$a_{4}$ & -1.8534 & -1.3485 \\
$a_{5}$ & 0.0114 & 0.0021 \\
\hline
\end{tabular}

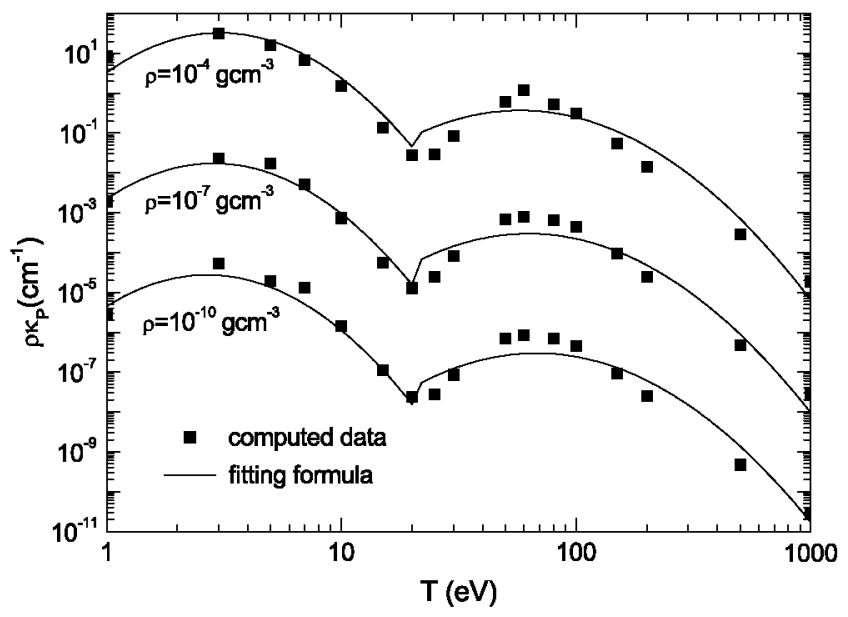

Fig. 7. Computed Planck mean opacity for carbon versus temperature at several densities (symbols) and fitting curves (solid) obtained by using Eq. (2).

\subsection{Theoretical study}

We have chosen an isothermal case $(40 \mathrm{eV})$ to study variations in density and the case of a fixed free electron number density $\left(10^{21} \mathrm{~cm}^{-3}\right)$ for the analysis of temperature dependence. Firstly, the optically thin situation is analyzed. For a given temperature, we
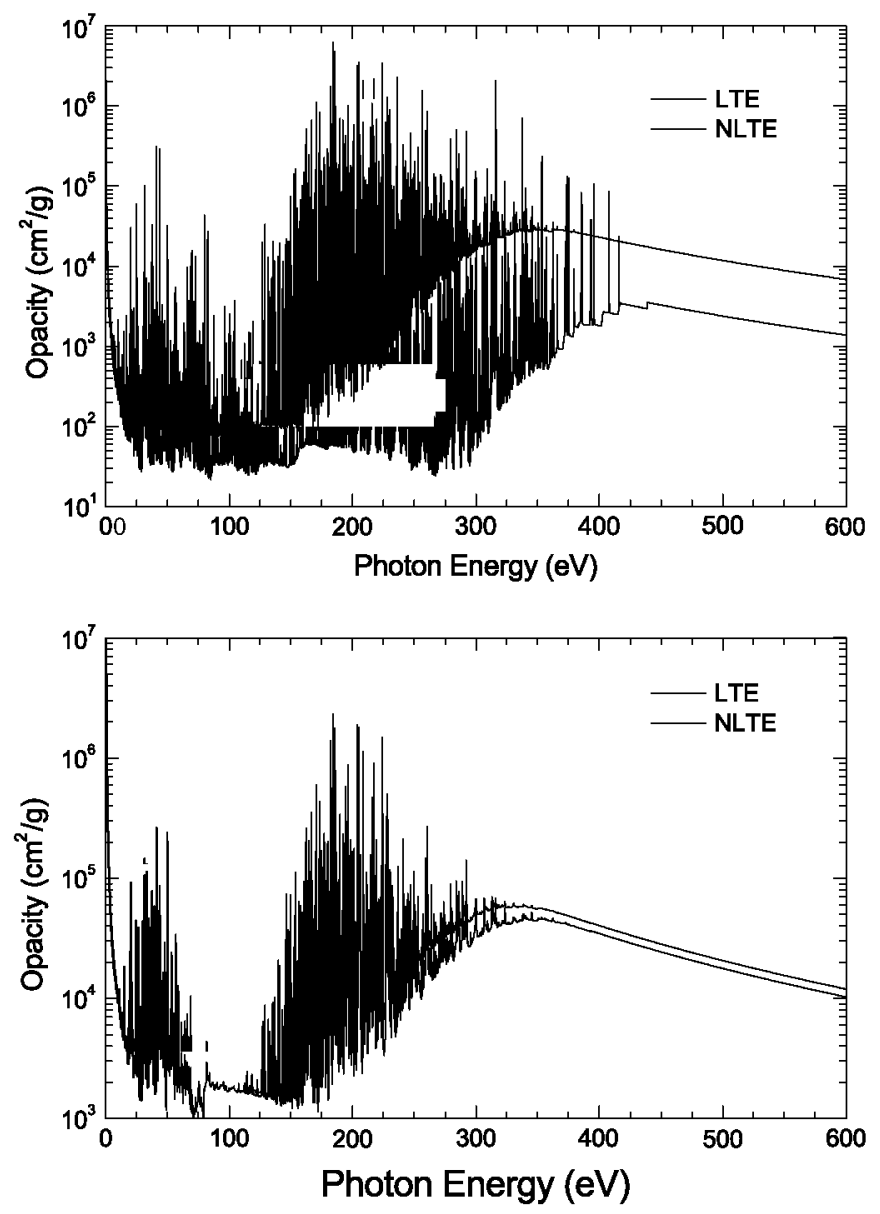

Fig. 8. Spectrally resolved opacity for an aluminum plasma at $\rho=10^{-4} \mathrm{~g} \mathrm{~cm}^{-3}$ (up) and $\rho=4 \times 10^{-3} \mathrm{~g} \mathrm{~cm}^{-3}$ (down) and a fixed temperature $T=40 \mathrm{eV}$. Differences can be observed when LTE is assumed. 

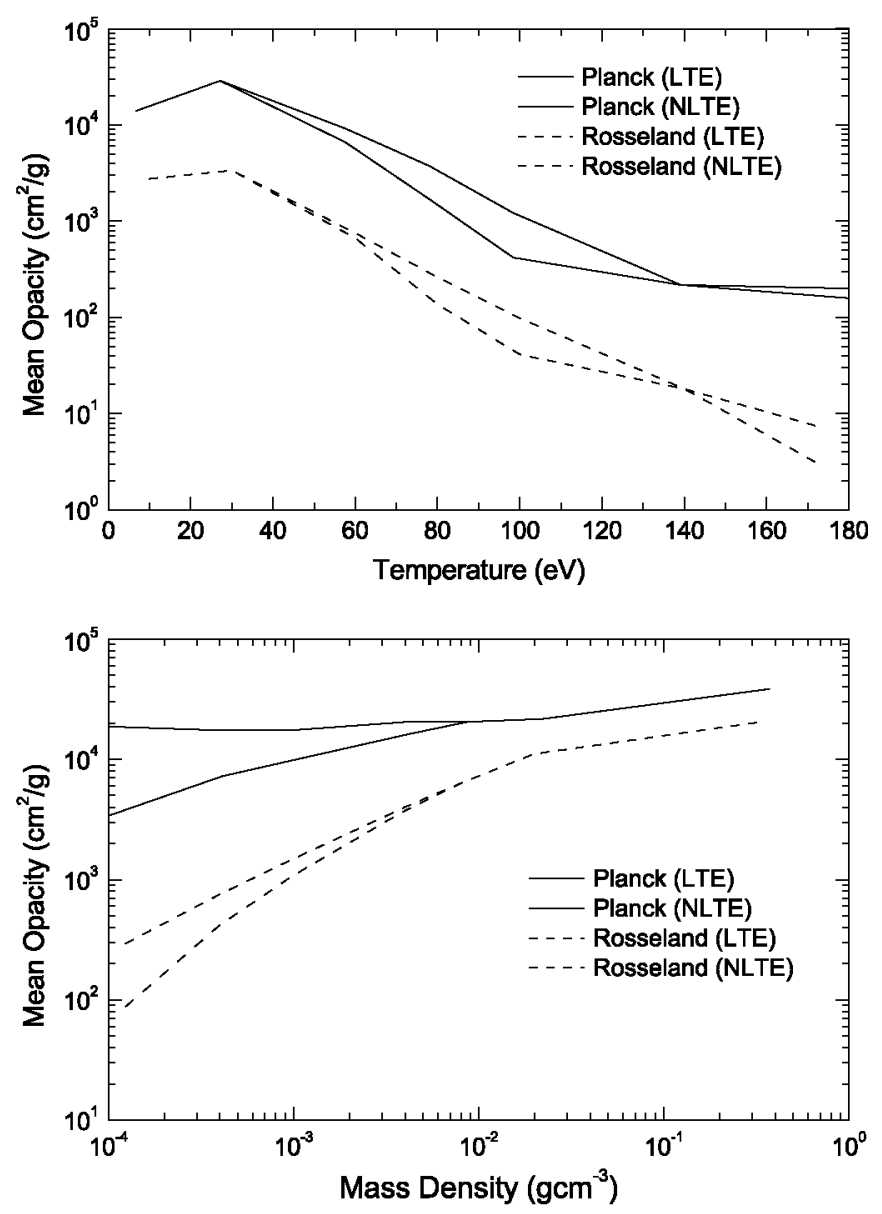

Fig. 9. Planck and Rosseland mean opacities of an aluminum plasma as a function of mass density at a fixed temperature $T=40 \mathrm{eV}$ (down) and as a function of temperature at a fixed electron density $n_{\mathrm{e}}=10^{21} \mathrm{~g} \mathrm{~cm}^{-3}$ (up). Differences can be observed when LTE is assumed.

observed that the spectrally resolved opacities increase with density (see Fig. 8), because of the decrease in average ionization.

Both Rosseland and Planck mean opacities increase with density (see Fig. 9 (down)). This increase is larger for the Rosseland mean opacity than for the Planck case. For this case the increase depends mainly on the differences in the position of the peaks but, in this sequence, they are rather small since there are little variations in the average ionization. On the other hand, Rosseland mean opacity also depends strongly on the line width. For a fixed temperature, the Doppler width remains constant, but the collisional width increases with density, which produces a larger change in the Rosseland mean opacity than the Planck one.

For a given density, the behavior with temperature is a little more complex. A shoulder is observed both in the Rosseland and Planck opacities (see Fig. 9 (up)). The increase of the mean opacities

Table 4

Rosseland $\left(\kappa_{\mathrm{R}}\right)$ and Planck $\left(\kappa_{\mathrm{P}}\right)$ mean opacities $\left(\mathrm{cm}^{2} / \mathrm{g}\right)$ for several densities $\left(\mathrm{g} \mathrm{cm}^{-3}\right)$ at $T=40 \mathrm{eV}$.

\begin{tabular}{lrrrrrr}
\hline$\rho$ & \multicolumn{1}{c}{$\kappa_{\mathrm{P}}^{\mathrm{ZY}}$} & \multicolumn{1}{c}{$\kappa_{\mathrm{P}}^{\mathrm{LTE}}$} & $\kappa_{\mathrm{P}}^{\mathrm{NLTE}}$ & \multicolumn{1}{c}{$\kappa_{\mathrm{R}}^{\mathrm{ZY}}$} & $\kappa_{\mathrm{R}}^{\mathrm{LTE}}$ & $\kappa_{\mathrm{R}}^{\mathrm{NLTE}}$ \\
\hline $1 \times 10^{-4}$ & 4236 & 3484 & 18540 & 68 & 66 & 248 \\
$4 \times 10^{-4}$ & 8130 & 7301 & 17214 & 316 & 419 & 755 \\
$1 \times 10^{-3}$ & 11380 & 10018 & 17449 & 845 & 1091 & 1494 \\
$4 \times 10^{-3}$ & 15900 & 16220 & 20375 & 3435 & 3746 & 4013 \\
\hline
\end{tabular}

LTE and NLTE values obtained with ABAKO/RAPCAL and those from Zeng and Yuan (ZY) [37] are displayed.
Table 5

Effects of the reabsorption on plasma average ionization $(\bar{Z})$, Planck $\left(\kappa_{\mathrm{P}}\right)$ and Rosseland $\left(\kappa_{R}\right)$ mean opacities $\left(\mathrm{cm}^{2} / \mathrm{g}\right)$ for several densities $\left(\mathrm{g} \mathrm{cm}^{-3}\right)$ and a fixed temperature of $T=40 \mathrm{eV}$.

\begin{tabular}{|c|c|c|c|c|c|}
\hline \multicolumn{3}{|c|}{$\rho=1 \times 10^{-4}$} & \multicolumn{3}{|c|}{$\rho=4 \times 10^{-3}$} \\
\hline $\bar{Z}$ & $\kappa_{\mathrm{P}}$ & $\kappa_{\mathrm{R}}$ & $\bar{Z}$ & $\kappa_{P}$ & $\kappa_{\mathrm{R}}$ \\
\hline \multicolumn{6}{|c|}{ Thin case } \\
\hline 7.555 & 18540 & 248 & 7.340 & 20375 & 4013 \\
\hline \multicolumn{6}{|c|}{$15-\mu \mathrm{m}$ thick slab } \\
\hline 7.609 & 17690 & 118 & 7.539 & 17507 & 3875 \\
\hline \multicolumn{6}{|c|}{$50-\mu \mathrm{m}$ thick slab } \\
\hline 7.687 & 16584 & 108 & 7.603 & 16867 & 3830 \\
\hline
\end{tabular}

at low temperatures is due to the fact that the maximum of the spectrally resolved opacity is approaching the maximum value of the Planck and its first derivative functions. For higher temperatures, the average ionization increases and, therefore, the mean opacities diminish. Great changes are observed in both mean opacities. These changes are basically due to the considerable shifts of the maximum values of the opacities toward higher photon energy because of the increase of the average ionization.

In Figs. 8 and 9 discrepancies are observed in the opacities when the plasma is not in LTE. They are appreciable even for near-LTE conditions as it happens in the case of Fig. 8 (down). Since SahaBoltzmann equations overestimate the average ionization with respect to the $\mathrm{CR}$ calculations, the mean opacities in the latter model are always greater than in the former. This is shown in Table 4 , where the results assuming LTE are given, and they have been compared with those obtained in LTE from other authors [37].

Changes in the opacities introduced by the plasma reabsorption are also determined. As is known, reabsorption produces an increase of the average ionization with respect to the optically thin situation. Therefore, both the Rosseland and Planck mean opacities will decrease (see Tables 5 and 6). Significant variations are observed between optically thin and thick situations, even when the modifications in the average ionization are small. Relative differences between optically thin and thick calculations of the mean opacities are weakly dependent on the temperature; however, the dependence with the density is more noticeable. The relative differences for the Planck case increase with the density and decrease with the temperature whereas the behavior for the Rosseland case is just the opposite. This can be understood by taking into account the dependence of the Rosseland and Planck mean opacities on the temperature and the density which was explained previously.

\subsection{K-shell spectroscopic diagnostics of a laser-produced aluminum plasma}

An experimental study to measure the opacity and emissivity of bound-bound transitions in laser-shocked dense and hot aluminum plasma was carried out at the LULI laser facility [38,39].

\section{Table 6}

Effects of the reabsorption on plasma average ionization $(\bar{Z})$, Planck $\left(\kappa_{\mathrm{P}}\right)$ and Rosseland $\left(\kappa_{\mathrm{R}}\right)$ mean opacities $\left(\mathrm{cm}^{2} / \mathrm{g}\right)$ for several temperatures $(\mathrm{eV})$ and a fixed density of $n_{\mathrm{e}}=10^{21} \mathrm{~cm}^{-3}$.

\begin{tabular}{|c|c|c|c|c|c|}
\hline \multicolumn{3}{|l|}{$T=80$} & \multicolumn{3}{|l|}{$T=120$} \\
\hline $\bar{Z}$ & $\kappa_{\mathrm{P}}$ & $\kappa_{\mathrm{R}}$ & $\bar{Z}$ & $\kappa_{\mathrm{P}}$ & $\kappa_{\mathrm{R}}$ \\
\hline \multicolumn{6}{|c|}{ Thin case } \\
\hline 10.266 & 3611 & 262 & 10.849 & 439 & 38 \\
\hline \multicolumn{6}{|c|}{$15-\mu \mathrm{m}$ thick slab } \\
\hline 10.505 & 2269 & 196 & 10.886 & 286 & 27 \\
\hline \multicolumn{6}{|c|}{$50-\mu \mathrm{m}$ thick slab } \\
\hline 10.543 & 2066 & 180 & 10.895 & 247 & 24 \\
\hline
\end{tabular}




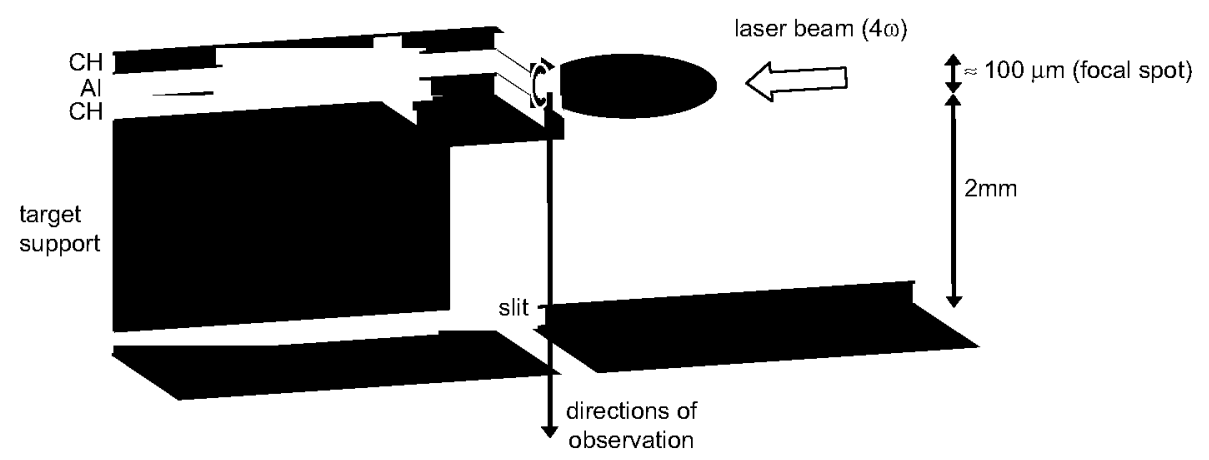

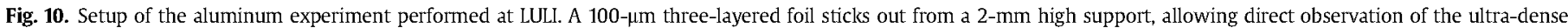
plasma region.

Theoretical investigations performed in parallel to these experiments obtained estimates of the opacity for He- and $\mathrm{H}$-like $\mathrm{Al}$ transitions such as Ly $\alpha, \operatorname{He} \beta$ and $\operatorname{He} \gamma$ for particular physical conditions. However, until now there had been no spectroscopic analysis including all the recorded emission features simultaneously or systematic study of the spatial dependence of the plasma physical conditions. Through a series of recent improvements in the ABAKO/RAPCAL code, we present in this work a preliminary determination of the electron temperature and density spatial profiles of such an aluminum plasma by the spectroscopic analysis of the recorded K-shell line emission spectra.

Fig. 10 shows a schematic picture of the experimental setup. The neodymium laser chain provided a 600 ps Gaussian pulse with a maximum energy of $80 \mathrm{~J}$ and a wavelength of $1053 \mathrm{~nm}$. A $4 \omega$ beam was focused onto a structured target, the $100 \mu \mathrm{m}$ focal spot yielded intensities as high as $2 \times 10^{14} \mathrm{~W} \mathrm{~cm}^{-2}$ irradiating the edge of a three-layered foil $(\mathrm{CH} / \mathrm{Al} / \mathrm{CH})$. The plastic was entirely converted into plasma during the laser shot, thus ensuring the aluminum plasma confinement. The laser-target interaction gives rise to a nick instead of a crater and this was the key to guarantee a rigorous tranversal observation of the ultra-dense plasma. In addition, this setup automatically produced a progressive spatial integration along the laser-target axis in the recorded film (see Fig. 11). Further details related to the experiment can be found in Refs. $[38,39]$.

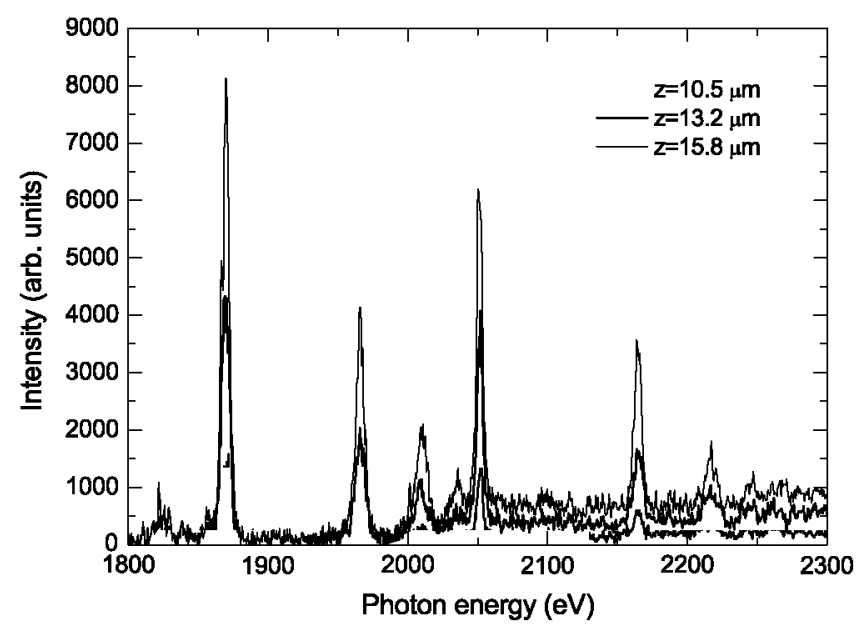

Fig. 11. Several extracted experimental lineouts. The spatial integration along targetlaser axis ( $z$-axis) given by the experimental setup can be observed, since each lineout lies below the following one.
For this particular application, ABAKO/RAPCAL computed a database of emergent intensities in the photon energy range from 1700 to $2400 \mathrm{eV}$ over a $20 \times 20$ grid of electron temperatures and densities in the domain of interest, i.e. $300-500 \mathrm{eV}$ and $10^{21}-10^{23} \mathrm{~cm}^{-3}$. For these calculations an optically $80-\mu \mathrm{m}$ thick aluminum plasma was assumed, which matches with the thickness of the aluminum layer of the target for the case analyzed here. To satisfy the required accuracy in the atomic data, we calculated them using FAC. We employed a semiempirical formula for estimating Stark widths [22]. Natural, Stark and Doppler broadenings were taken into account in the context of Voigt line profiles. Complete redistribution was assumed and line overlapping considered. Finally, the extraction of the electron temperature and density for a given spectral lineout is performed by searching in the database the synthetic spectrum that yields the best fit to the data, in the sense of a least square minimization. An example is given in Fig. 12. A systematic application of this procedure to each of the lineouts in the recorded film results in a spatial profile of $T_{e}$ and $n_{e}$.

According to the analysis described above, Fig. 13 shows the inferred electron temperature and density spatial profiles. As was expected the density peaks at points near the target. The average temperature is about $430 \mathrm{eV}$, showing some oscillations in a $50-\mathrm{eV}$ width interval. This is because only the first $65 \mu \mathrm{m}$ of the plasma was analyzed and we should expect temperatures to drop at points far from the target.

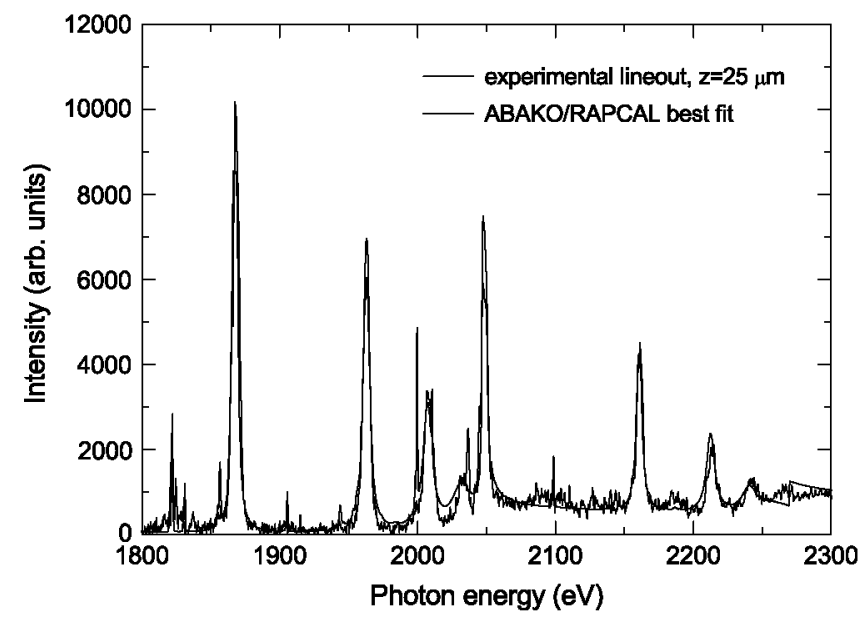

Fig. 12. An example of $A B A K O / R A P C A L$ best fit for an aluminum K-shell emission spectrum. The analysis gives $n_{\mathrm{e}}=1.52 \times 10^{22} \mathrm{~cm}^{-3}$ and $T=417 \mathrm{eV}$. 

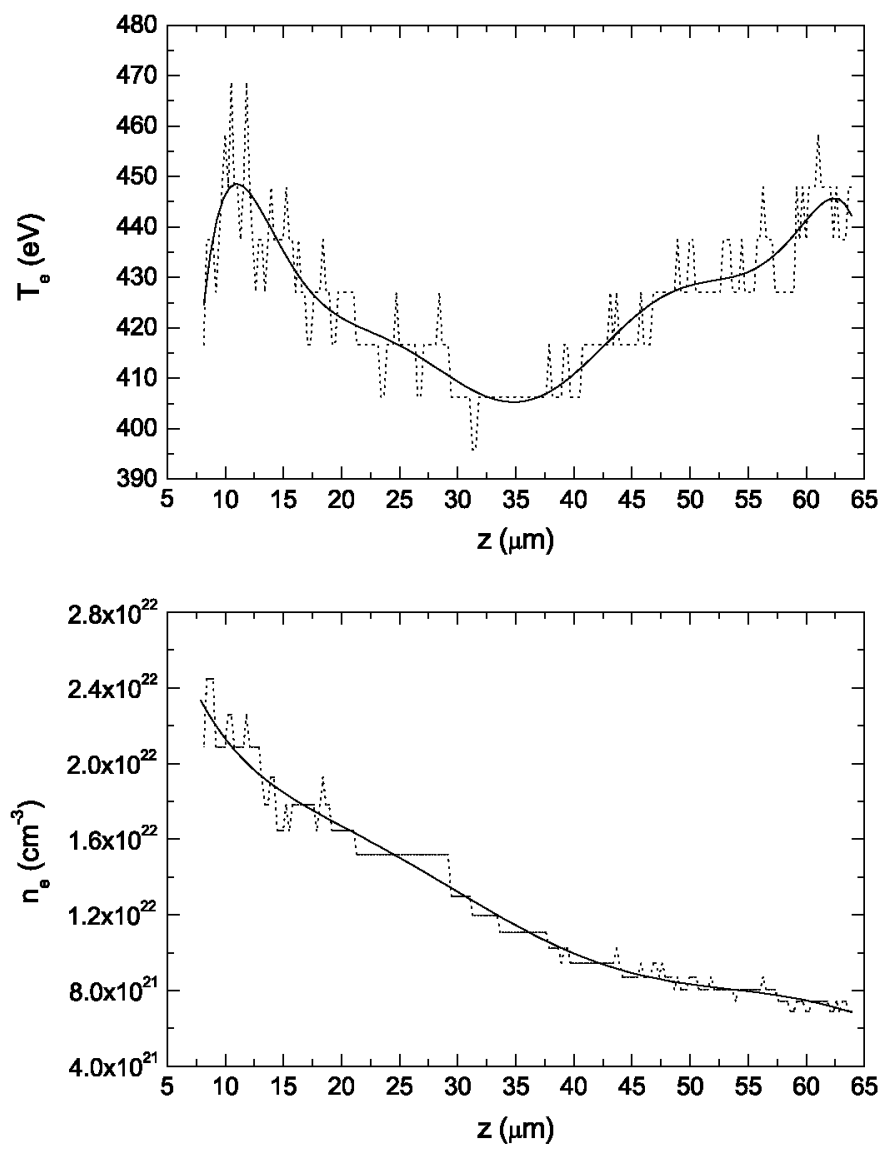

Fig. 13. Inferred spatial profiles of temperature (up) and density (down). The $z$-axis represents the target-laser axis. For clarity in the interpretation smoothing polynomials have been added over the point-by-point diagnosed values.

\section{Conclusions}

In this work the flexible and modular set of codes ABAKO/ RAPCAL has been shown to be useful in obtaining estimates of optical properties for optically thin and thick plasmas over a wide range of densities and temperatures.

Atomic structure, levels, energies, oscillator strengths and others' atomic data needed for solving the $\mathrm{CR}$ matrix can be determined by two ways. The original one uses analytical potentials in the relativistic DCA approach with a considerable computational time saving. This procedure provides satisfactory results as has been shown by means of comparison with other models. The second one, which uses more accurate and detailed atomic structure, can be provided by an external computer code or an atomic database. The first option is very useful when we are interested in providing opacities and emissivities required for hydrocodes and, therefore, speed of computation is desired although some accuracy is lost. However, when an experimental study is involved and a spectroscopic analysis is required, the second option is then used.

In any case, the sparse matrix technique is used for the storage of the $\mathrm{CR}$ matrix elements. Also, the problem often involves very large sparse matrices, so iterative methods are used to carry out the matrix inversion because they typically require less memory and they can yield an approximation to the solution significantly faster than a direct method.

Both thin and thick plasmas can be analyzed with this set of codes. The bound-bound self-absorption effects in homogeneous plasmas are modeled using the escape factor formalism for planar, cylindrical, and spherical geometries.
To know the goal and possibilities of this set of codes, results for ion populations, plasma average ionization, spectrally resolved opacities and emissivities, mean opacities, and modeling of experimental spectra are shown in this work. Thus, a variety of calculations for carbon and aluminum plasmas have been presented, both in LTE and NLTE situations and for a wide range of plasma conditions. Furthermore, the opacity effects on the ion populations and the radiative properties for an optically thick homogeneous plasma have been briefly studied.

Comparisons with theoretical or experimental data provided show that this set of codes combine accuracy and speed and they can be a valid tool for a large number of applications. For instance, large-scale calculations could be used as input for hydrocodes and more specific implementations of the codes current version could be helpful in experimental modeling and to target design for inertial fusion.

Finally, using this set of codes, Rosseland and Planck mean opacities have been computed with the main goal of fitting an NLTE analytical expression for mean opacities that depend on plasma temperature and density. In this work, parameters of this analytical expression for optically thin carbon plasmas have been provided, and a more elaborate work is now under way, extending the methodology to other elements.

\section{Acknowledgements}

Authors acknowledge Prof. Rose for his valuable comments and suggestions in improving the paper. This work has been supported by a Research Project of the Spanish Ministry of Science and Innovation ENE2008-06668-C02/FTN and also by the "Keep in Touch" Project of the EU.

\section{References}

[1] S.B. Hansen, K.B. Fournier, C. Bauche-Arnoult, J. Bauche, O. Peyrusse, A comparison of detailed level and superconfiguration models of neon, J. Quant. Spectrosc. Radiat. Transfer 99 (2006) 272-282.

[2] O. Peyrusse, Complex atom physics and radiative properties of hot dense plasmas, Nucl. Fusion 44 (2004) S202-S207.

[3] T.S. Perry, S.J. Davidson, F.J.D. Serduke, D.R. Bach, C.C. Smith, J.M. Foster RJ. Doyas, R.A. Ward, C.A. Iglesias, F.J. Rogers, J. Abdallah Jr., R.E. Stewart, J.D. Kilkenny, R.W. Lee, Opacity measurements in a hot dense medium, Phys. Rev. Lett. 67 (1991) 3784-3787.

[4] C.A. Iglesias, M.H. Chen, D.L. McWilliams, J.K. Nash, F.J. Rogers, Observations on radiation transfer experiments using K-shell absorption spectra, J. Quant Spectrosc. Radiat. Transfer 54 (1995) 185-193.

[5] G. Winhart, K. Eidmann, C.A. Iglesias, A. Bar-Shalom, E. Mínguez, A. Rickert S.J. Rose, XUV opacity measurements and comparison with models, J. Quant. Spectrosc. Radiat. Transfer 54 (1995) 437-446.

[6] C. Chenais-Popovics, F. Gilleron, M. Fajardo, H. Merdji, T. Mi Balla, J.C. Gauthier, P. Renaudin, S. Gary, J. Bruneau, F. Perrot, T. Blenski, W. Fölsner, K. Eidmann, Radiative heating of $\mathrm{B}, \mathrm{Al}$ and $\mathrm{Ni}$ thin foils at $15-25 \mathrm{eV}$ temperatures, J. Quant. Spectrosc. Radiat. Transfer 65 (2000) 117-133.

[7] R. Florido, J.M. Gil, R. Rodríguez, J.G. Rubiano, P. Martel, E. Minguez, Line photon transport in a non-homogeneous plasma using radiative coupling coefficients, J. Phys. IV 133 (2006) 993-996.

[8] R. Florido, R. Rodríguez, J.M. Gil, J.G. Rubiano, P. Martel, D. Suárez, M. Mendoza, E. Mínguez, ABAKO: a new code for population kinetics and radiative properties of plasmas under NLTE conditions, J. Phys.: Conf. Ser. 112 (2008) 042008

[9] R. Rodríguez, R. Florido, J.M. Gil, J.G. Rubiano, P. Martel, E. Mínguez, RAPCAL code: a flexible package to compute radiative properties for optically thin and thick low and high- $Z$ plasmas in a wide range of density and temperature, Laser Part. Beams 26 (2008) 433-448.

[10] P. Martel, L. Doreste, E. Mínguez, J.M. Gil, A parametric potential for ions from helium to iron isoelectronic sequences, J. Quant. Spectrosc. Radiat. Transfer 54 (1995) 621-636.

[11] P. Martel, J.G. Rubiano, J.M. Gil, L. Doreste, E. Mínguez, Analytical expressions for the $n$-order momenta of charge distribution for ions, J. Quant. Spectrosc Radiat. Transfer 60 (1998) 623-633.

[12] J.M. Gil, P. Martel, E. Mínguez, J.G. Rubiano, R. Rodríguez, F.H. Ruano, An effective analytical potential including plasma effects, J. Quant. Spectrosc Radiat. Transfer 75 (2002) 539-557. 
[13] R. Rodríguez, J.G. Rubiano, J.M. Gil, P. Martel, E. Mínguez, R. Florido, Development of an analytical potential to include excited configurations, J. Quant. Spectrosc. Radiat. Transfer 75 (2002) 723-739.

[14] J.G. Rubiano, R. Rodríguez, J.M. Gil, F.H. Ruano, P. Martel, E. Mínguez, A screened hydrogenic model using analytical potentials, J. Quant. Spectrosc. Radiat. Transfer 72 (2002) 575-588.

[15] M.F. Gu, The flexible atomic code, in: 14th APS Topical Conference on Atomic Processes in Plasmas, AIP, 2004, pp. 127-136.<http://kipac-tree.stanford.edu/ facl>.

[16] W. Lotz, An empirical formula for the electron-impact ionization cross-section, Z. Phys. 206 (1967) 205-211.

[17] H. Van Regemorter, Rate of collisional excitation in stellar atmospheres, Astrophys. J. 136 (1962) 906-915.

[18] R.D. Cowan, The Theory of Atomic Structure and Spectra, University of California Press, 1981. $\mathrm{ftp}: / /$ aphysics.lanl.gov/pub/cowan/>.

[19] H.R. Griem, Principles of Plasma Spectroscopy, Cambridge University Press, 1997.

[20] J.C. Stewart, K.D. Pyatt, Lowering of ionization potentials in plasmas, Astrophys. J. 144 (1966) 1203-1211.

[21] R. Rodríguez, J.M. Gil, R. Florido, J.G. Rubiano, P. Martel, E. Mínguez, Code to calculate optical properties for plasmas in a wide range of densities, J. Phys. IV 133 (2006) 981-984.

[22] M.S. Dimitrijevic, N. Konjevic, Simple estimates for Stark-broadening of ion lines in stellar plasmas, Astron. Astrophys. 172 (1987) 345-349.

[23] S.J. Rose, Calculations of the radiative opacity of laser-produced plasmas, J. Phys. B: At. Mol. Opt. Phys. 25 (1992) 1667-1681.

[24] C.H. Skinner, G. Federici, Is carbon a realistic choice for ITER's divertor? Phys. Scr. T124 (2006) 18-22.

[25] J. Filevich, J. Grava, M. Purvis, M.C. Marconi, JJ. Rocca, J. Nilsen, J. Dunn, W.R. Johnson, Multiply ionized carbon plasmas with index of refraction greater than one, Laser Part. Beams 25 (2007) 47-51.

[26] J.L. Weaver, M. Busquet, D.G. Colombant, A.N. Mostovych, U. Feldman, M. Klapisch, J.F. Seely, C. Brown, G. Holland, Experimental benchmark for an improved simulation of absolute soft-X-ray emission from polystyrene targets irradiated with the Nike laser, Phys. Rev. Lett. 94 (2005) 045002.

[27] J. Colgan, C.J. Fontes, J. Abdallah Jr., Collisional-radiative studies of carbon plasmas, High Energy Density Phys. 2 (2006) 90-96.
[28] M. Poirier, F. de Gaufridy de Dortan, A comparison between detailed and configuration-averaged collisional-radiative codes applied to nonlocal thermal equilibrium plasmas, J. Appl. Phys. 101 (2007) 063308.

[29] M. Poirier, On various validity criteria for the configuration average in collisional-radiative codes, J. Phys. B: At. Mol. Opt. Phys. 41 (2008) 025701.

[30] J.M. Gil, R. Rodríguez, R. Florido, J.G. Rubiano, P. Martel, E. Mínguez, Determination of Corona, LTE and NLTE regimes of optically thin carbon plasmas, Laser Part. Beams 26 (2008) 21-31.

[31] N.H. Magee, J. Abdallah Jr., J. Colgan, P. Hakel, D.P. Kilcrease, S. Mazevet, M. Sherrill, C.J. Fontes, H.L. Zhang, Los Alamos Opacities: Transition from LEDCOP to ATOMIC, in: 14th APS Topical Conference on Atomic Processes in Plasmas, AIP, 2004, pp. 168-179.

[32] G.D. Tsakiris, K. Eidmann, An approximate method for calculating Planck and Rosseland mean opacities in hot, dense plasmas, J. Quant. Spectrosc. Radiat. Transfer 38 (1987) 353-368.

[33] J. Lindl, Development of the indirect-drive approach to inertial confinement fusion and the target physics basis for ignition and gain, Phys. Plasmas 2 (1995) 3933-4024.

[34] E. Mínguez, R. Muñoz, R. Ruiz, R. Yagüe, Analytical opacity formulas for ICF elements, Laser Part. Beams 17 (1999) 799-806.

[35] E. Mínguez, R. Ruiz, P. Martel, J.M. Gil, J.G. Rubiano, R. Rodríguez, Scaling law of radiative opacities for ICF elements, Nucl. Instrum. Methods Phys. Res., Sect. A 464 (2001) 218-224.

[36] E. Mínguez, P. Martel, J.M. Gil, J.G. Rubiano, R. Rodríguez, Analytical opacity formulas for ICF elements, Fusion Eng. Des. 60 (2002) 17-25.

[37] J. Zeng, J. Yuan, Detailed-term-accounting approximation calculations of the radiative opacity of aluminum plasmas: a systematic study, Phys. Rev. E 66 (2002) 016401

[38] R. Schott, F. Philippe, P. Angelo, E. Dufour, A. Poquerusse, E. LeboucherDalimier, P. Sauvan, P. Velarde, F. Ogando, E. Mînguez, J.M. Gil, J.G. Rubiano, R. Rodríguez, P. Martel, R. Mancini, Access to spectrally resolved ultra-dense hot low $z$ emissivities and opacities, , In: 16th International Conference on Spectral Line Shapes, vol. 12, American Institute of Physics, 2002, pp. 340-51.

[39] R. Schott, F. Philippe, P. Angelo, E. Dufour, A. Poquerusse, E. Dalimier, P. Sauvan, E. Mínguez, J.M. Gil, J.G. Rubiano, R. Rodríguez, P. Martel, R. Mancini, Low Z opacities at high densities, J. Quant. Spectrosc. Radiat. Transfer 81 (2003) 441-450. 